\title{
A step toward GPS/INS personal navigation systems: real-time assessment of gait by foot inertial sensing*
}

\author{
Filippo Cavallo, Angelo M. Sabatini, Vincenzo Genovese \\ ARTS Lab - Advanced Robotics Technology \& Systems Lab \\ Scuola Superiore Sant'Anna \\ Pisa, Italy \\ \{F.Cavallo, A.Sabatini, V.Genovese\}@mail.arts.sssup.it
}

\begin{abstract}
In this paper we develop a system for which applications in the field of personal navigation are planned. In the current version, the system embodies a global positioning system (GPS) receiver and an inertial measurement unit (IMU), composed of two dual-axis accelerometers and one single-axis gyro. The IMU is positioned at a subject's foot instep, and it is intended to produce estimates of some gait parameters, including stride length, stride time, and walking speed. Data from GPS and IMU are managed by a DSP-based control box.

The computations performed by the DSP processor allow to detect subsequent foot contacts by a threshold-based method applied to gyro signal, and to reconstruct the trajectory of the foot instep by numerical strapdown integration. Features of human walking dynamics are incorporated in the algorithm to enhance the estimation accuracy against errors due to sensor noise and integration drift. All computations are performed by the DSP processor in real-time conditions.

The foot sensor performance is assessed during outdoor level walking trials. The traveled distance estimated by inertial deadreckoning is compared with the estimate produced by GPS in experimental conditions where GPS can be used as a reference source for accurate absolute positioning. Results show the remarkable accuracy achieved by foot inertial sensing.
\end{abstract}

Index Terms - inertial sensing, GPS, personal navigation, strapdown integration, gait assessment.

\section{INTRODUCTION}

At present, human motor performance can be accurately assessed using several tools. The most important technology used to detect and track human body motion is video motionsensing, i.e., optoelectronic measurement systems. However, the use of these systems is critical because they are expensive and difficult to operate outside the limits of controlled laboratory settings, where the human behavior is known to be constrained and influenced to a great extent. Because of this, the interest for ambulatory monitoring systems is rapidly increasing.

From the clinical viewpoint, ambulatory monitoring systems are interesting for the opportunity they provide, in principle, to expand the range of environments, including daily-life environments, where a person's level of functional ability can be quantitatively studied; on the other hand, the ability to locate a moving person is of great importance in many other applications, including electronic travel aids for the blind or visually impaired, personal navigation systems, and, in a broader sense, context-aware wearable systems. In order to circumvent the limitations of video-motion sensing, inertial sensing technology is actively researched as a suitable approach to assess human motor performance and track human body motion in unrestrained daily-life conditions, which gives rise to a quite novel view of inertial sensors as devices useful for functional and navigational tasks [1].

Until recent years, inertial sensors have only found use to monitor the motion of man-made vehicles, including spaceships, planes, ships, submarines, cars, and, more recently, wheeled and legged robots. Recent advances in microelectromechanical systems (MEMS) technologies have led to the development of a new generation of inertial sensors with features (size, weight, power consumption, cost) which are quite useful in the present context. Body-mounted inertial sensors make it possible to determine position and orientation information based on the measurement of physical quantities (acceleration, angular velocity) which are directly related to the motion of the body part where they are positioned. In inertial navigation systems (INSs), the main problem is that position and orientation are found by time-integrating the signals from accelerometers and gyros, including any sensor drift and noise superimposed to them (dead-reckoning navigation). As a result, position and orientation errors tend to grow unbounded, and it is generally believed that double integration of acceleration signals is not accurate enough for long term monitoring of human motion [2]. Apparently, the problem of absolute positioning in outdoor environments is easily solved by using an externally referenced sensing technology such as Global Positioning System (GPS). However, the disadvantages with GPS - inability to work in indoors and unavailability of satellite signals in environments such as urban canyons - suggest that the combined use of dead-reckoning based on inertial sensing with GPS would be a better answer to our positioning needs.

Most previous works in the field of personal navigation systems revolve around the integration of inertial sensing and GPS. A GPS receiver with a centimetric precision using phase differential positioning and operated at high sampling rates is used, in combination with a tri-axis accelerometer mounted at the subject's waist, to produce accurate estimates of the vertical displacement of the trunk during walking [3].

\footnotetext{
* This work is partially supported by funds from the Italian Ministry of University and Research.
} 
Other applications of personal navigation systems are, in a sense, more traditional [4]-[6]: these involve the use of GPS as an external aid to estimate the biases inertial sensors are affected with, in the attempt to improve the accuracy of the dead-reckoning method during GPS outages. Kalman filters (KFs) are the analytical tool typically used to perform the sensor integration task [7].

There appears to be something peculiar to the way humans walk that can be exploited to improve the performance of GPS/INS approaches to personal navigation. The key problem is to find a method to measure length and direction of displacement using step time as the basic unit of time, so as to accurately determine the distance and heading from a known origin. Detecting step occurrences can be based on accelerometers or gyroscopes positioned in different parts of the body, such as lower trunk, waist, thigh, shank, heel, foot [8]-[11]. A simple model-based approach to the problem of estimating step length hypothesizes that, once a method is available to determine step time, step length estimation can be based on cadence. In [4] it is suggested that the step length could be estimated online based on a linear relationship between measured cadence and step length, whose validity, discussed in [5], is however limited to level walking in open spaces. The approach in [5] exploits the relationship existing between walking speed and the root mean square (RMS) values of waist $3 \mathrm{D}$-accelerations, starting from the premise that the main goal of locomotion is to promote the body centre of mass (BCOM) displacement in space. Unfortunately, intraindividual physiological variability and environmental conditions heavily influence the accuracy of the relationships exploited to infer the quantities of interest. Hence, frequent calibration procedures may be needed, which requires additional sensors [12].

In this paper we intend to move a step in the direction of implementing a GPS/INS personal navigation system whose main feature is the implementation of a direct method for determining the traveled distance. Direct method means that we deal directly with the strapdown integration from an inertial measurement unit (IMU), which is positioned at subject's foot instep [13]. The gyro signal is used to perform the gait phase segmentation. Cyclical gait features are used to overcome the problems of integrating sensor noise and drifts, provided that the initial conditions for the time-integrals (alignment) are determined using the redundancy of information on foot motion during the foot-flat phase of the walking cycle. All computations (gait phase segmentation, integration, and drift compensation) are carried in real-time conditions.

In this paper, we are not interested in the design of filtering algorithms to fuse inertial dead-reckoning with absolute positioning by GPS, neither in determining the direction of displacement by, e.g., gyro-compassing methods. We intend to assess the foot sensor performance during outdoor level walking trials. The traveled distance estimated by inertial dead-reckoning is compared with the estimate produced by GPS in experimental conditions where GPS can be used as a reference source for accurate absolute positioning. Results show the remarkable accuracy achieved by foot inertial sensing.

\section{METHODS}

\section{A. Instrumentation}

The developed GPS/INS prototype consists of an IMU, a GPS receiver and a control box embedding a DSP board.

The IMU is composed of two dual-axis piezoresistive accelerometers (Analog Device ADXL210E), arranged perpendicular to one another so as to form a tri-axis accelerometer (actually two sensitive axes turn out to be parallel by sensor construction), and one single-axis piezoelectric gyro (Murata ENC-03J), Fig. 1.

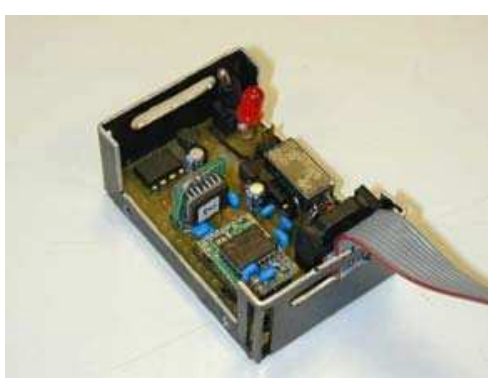

Fig. 1 The developed IMU, endowed with two dual-axis accelerometers and one single-axis gyro.

The accelerometers measure accelerations over a fullscale range of $\pm 10 \mathrm{~g}\left(\mathrm{~g}=9.81 \mathrm{~m} / \mathrm{s}^{2}\right.$ is the gravity acceleration) - the device sensitivity is $K_{a}=0.1 \mathrm{~V} / g$. The gyro exploits the Coriolis acceleration effect to measure the angular velocity around the sensor longitudinal axis. The full-scale range is in excess of $\pm 300 \mathrm{deg} / \mathrm{s}$ - the device sensitivity is $K_{g}=0.67 \mathrm{mV} / \mathrm{deg} / \mathrm{s}$.

The IMU is attached to the subject's (right) foot instep, using a Velcro strap so as to snugly fix the unit to the shoe. Before testing, in-field calibration procedures are performed for either accelerometers or gyro [14]. Particular care is for positioning of the accelerometers: we want that two sensitive axes of the tri-axis accelerometer are approximately parallel to the sagittal plane, the third sensitive axis is oriented in the medial-lateral direction, parallel to the gyro sensitive axis (by sensor construction). Before each walking trial, the pitch and roll angles are checked by gravimetric tilt sensing. This is done to detect significant movements of the IMU relative to the foot throughout the experimental session.

The communication system of the GPS receiver (GARMIN GPS 35-HVS) is based on the NMEA 0183 ASCII interface protocol. The messages from the GPS receiver we are interested contain a wealth of information, such as the geographic coordinates of the antenna location (latitude and longitude), the antenna height relative to mean sea level, the number of satellites involved in the computation of these data, and, finally, the horizontal dilution of precision (HDOP) - a quantitative indication of the reliability of GPS measurements.

The control box, shown in Fig. 2, encompasses a TMS320F243 evaluation module, a standalone board that 
includes the Texas F243 digital signal processor (DSP). The control box accomplishes three main functions: to parse and interpret the NMEA strings; to convert geographic coordinates into local ground coordinates (North-East); to implement the signal processing functions which are needed for real-time parameterization of gait.

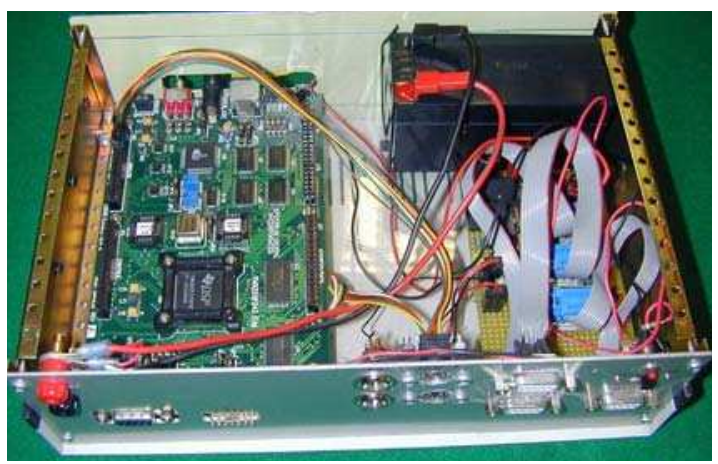

Fig. 2 DSP-based control box

\section{B. Real-time data processing}

The gait parameterization is performed by the DSP-based control box, Fig. 3. The real-time processing software is written in $\mathrm{C}$ programming language. The code can be divided in three modules: the module which implements the strapdown integration from inertial data; the module which parses the NMEA strings from the GPS receiver and computes the local ground coordinates; the module which implements the clock reference used to perform integration and time synchronization between GPS and IMU operation.

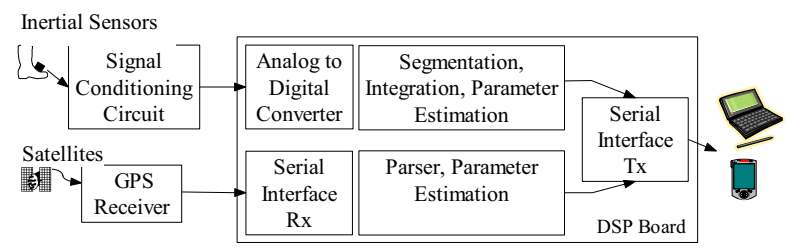

Fig. 3 Software structure in C programming language implemented in the DSP-based control box.

The accelerometers' and gyroscope signals are submitted to Analog-to-Digital conversion with 10 quantization bit at a rate $f_{s}=200 \mathrm{~Hz}$. The acquired data are filtered using a numerical fourth-order low-pass Butterworth filter (cut-off frequency: $7.5 \mathrm{~Hz}$ ).

The strapdown integration technique exploits the cyclical properties of gait, so as to process the foot sensor signals and to prevent the unbounded growth of errors due to lowfrequency noise, i.e., offset and sensitivity drift [13]. The initial condition of the foot orientation in the sagittal plane, relative to a ground-based Cartesian coordinate system, is obtained by processing the accelerometer signals during the foot-flat phase, when the accelerometers can be used to perform gravimetric tilt sensing.

The gait phase segmentation procedure is driven by the gyro signal through the detection of different phases, including heel-off, toe-off, heel-strike, foot-flat. We briefly describe how the heel-off and foot-flat detections take place. More details about the operation of the gait phase segmentation procedure are reported elsewhere [13]-[14].

Suppose that the initial state occupied by the subject is ST, i.e. the subject is standing still in the upright posture and the foot is at rest. While in the ST state, the finite-state algorithm waits for the transition to the heel-off phase, to determine the heel-off time. It is assumed that this transition occurs when the angular velocity magnitude $\omega$ is less than a specified threshold value $\lambda_{\mathrm{HO}}$ during a backward-search from the first (negative) local minimum occurring at the toe-off time. The heel-off time denotes the time instant $T_{\text {start }}$ for the signal integration to start, Fig. 4. The foot-flat event, corresponding to when the foot is flat on the ground the first time, is assumed to be detected when $\omega<\lambda_{\mathrm{FF}}\left(\lambda_{\mathrm{FF}}\right.$ is a specified threshold value $)$ - the angular velocity is almost steady at $0 \mathrm{deg} / \mathrm{s}$ when the foot is at rest during a forward search from the second (negative) minimum occurring at the heel-strike time. The foot-flat time denotes the time instant $T_{\text {end }}$ for the signal integration to end, Fig. 4.

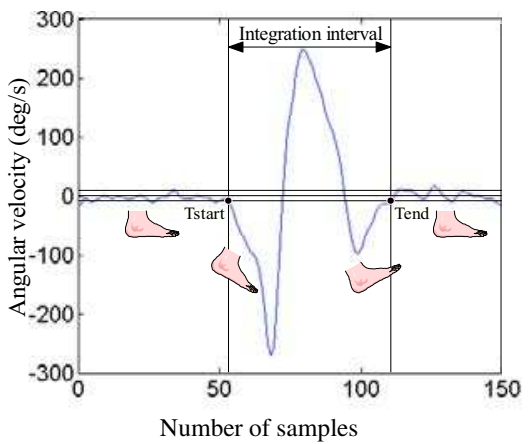

Fig. 4 Identification of the interval for strapdown integration.

The threshold values are selected empirically: $\lambda_{\mathrm{HO}}=\lambda_{\mathrm{FF}}=$ $15 \mathrm{deg} / \mathrm{s}$. These settings yield a satisfactory trade-off between false alarms and missed detections and work reliably for the experimental conditions described in this paper.

In the approach to strapdown integration described in this paper, the change of foot orientation estimate relies on the gyro when the leg is flying in space; the accelerometer is used as an absolute orientation measuring device when the foot is at rest. A static tri-axis accelerometer provides information about the inclination, however it cannot provide information concerning rotation around the vertical (yaw). The assumption that the foot movement occurs in the sagittal plane allows to relieve this difficulty of gravimetric tilt sensing [13].

The foot orientation estimate is used twice during the localization estimation. First, the accelerometer measures both the body's own acceleration $a_{\text {acc }}$ as the superposition of the sensed acceleration in the body frame, and the projection of the gravitational acceleration on the body frame. The next step requires double-integration of $a_{\text {acc }}$, once again projected in the reference frame using the calculated foot sagittal orientation, to derive the position. The integration method is based on the trapezoidal rule. 
Since the gait features are cyclical, the foot inclination angle during stance is approximately constant from one gait cycle to the next gait cycle. Hence, to prevent accumulation of offset and sensitivity drift errors in the integral of the gyro signal, a resetting mechanism is applied, by compensating the drift using the initial and end conditions for a stride. The same approach can be applied to the result of the first integration of the acceleration signal, again imposing the initial and end conditions for a stride to avoid drift (null velocity) [13].

The GPS receiver continuously transmits the NMEA sentences to the DSP-based control box via the available serial port. A conversion equation is needed to relate geodetic coordinates (latitude and longitude) to local ground coordinates (North and East, in meters) [15]. For a particular location on the Earth's surface, we want to know how many meters of north-south or east-west travel correspond to how many degrees of latitude or longitude, respectively. Note that, if we assume that Earth is a perfect ellipsoid with a fixed equatorial radius and eccentricity, the conversion factors depend on current latitude $\phi$. Under the additional assumption of short displacements, we can consider that movement occurs in a plane tangential to the ellipsoid at the current location. The conversion constants between meters and degrees are then:

$$
\begin{aligned}
& C_{1}=\frac{1}{32.5572\left(1-0.0066934 \cdot \sin ^{2} \phi\right)^{3 / 2}} \\
& C_{2}=\frac{\cos \phi}{33.339284\left(1-0.0066934 \cdot \sin ^{2} \phi\right)^{1 / 2}}
\end{aligned}
$$

where $C_{1}$ and $C_{2}$ are, respectively, the distance in meters north to south and east to west, respectively - corresponding to the change of one arc second in latitude and longitude.

Another problem with data acquisition is the lack of synchronization between IMU and GPS. While GPS data are provided at a rate of one reading per second, the IMU delivers the estimated length of the last stride and the associated time stamp as soon as the integration, applied to gravitycompensated acceleration sequences, is terminated. The time instants when the parameter estimates are delivered are not the same as the time instants when the latest values of the local ground coordinates are ready. A reference clock is then implemented in the DSP-based control box, which provides the time stamps for both piece of data starting from a known time origin common to both.

\section{Testing}

The experimental sessions were performed by asking one subject to walk for tracks of several hundred meters in a track and field stadium. The experiments were repeated in the same fashion in different days, so as to test for possible influence of environmental conditions on the system performance.

Each trial consisted of walking for a $400 \mathrm{~m}$ track. In order to analyse gait in steady-state conditions, the subject started with walking 10-15 m before the start point of each track and went beyond the end point by three or four additional strides. The subject had a single switch input device in his hand, which was used to temporally mark several checkpoints along the track. Five checkpoints along each track were annotated: start point, $120 \mathrm{~m}, 200 \mathrm{~m}, 320 \mathrm{~m}$, end point. Every time the subject went beyond a checkpoint, the event was annotated in a log file by pushing the single switch. The subject was also asked to adopt a uniform rhythm of walking; this was made easier by providing a metronome beep. The selected cadences were $45,50,55$ and 60 strides $/ \mathrm{min}$.

The GPS receiver was positioned on the subject's waist. Other electronic devices, including the $12 \mathrm{~V}$-battery and the control box, were placed in a rucksack. All data - local ground coordinates from GPS, gait spatio-temporal parameters from IMU - stride length and time - were transmitted via a standard serial communication interface (RS232 protocol) to a notebook, for immediate display. Additionally, the notebook, firmly anchored to the subject's waist, saved data from each experimental session.

\section{RESUltS}

Table 1 reports a summary of results achieved with the developed IMU. The walking speed at each gait cycle is obtained by dividing stride length by stride time.

TABLE 1

Summary of results achieved for gait parameters estimated by IMU, mean \pm standard deviation (SD).

\begin{tabular}{|c|c|c|c|c|}
\hline Cadence [stride/min] & 45 & 50 & 55 & 60 \\
\hline Stride length $[\mathrm{m}]$ & $1.16 \pm 0.06$ & $1.27 \pm 0.04$ & $1.33 \pm 0.05$ & $1.38 \pm 0.04$ \\
\hline Stride time $[\mathrm{s}]$ & $1.33 \pm 0.08$ & $1.20 \pm 0.08$ & $1.09 \pm 0.08$ & $1.04 \pm 0.09$ \\
\hline Walking speed $[\mathrm{m} / \mathrm{s}]$ & $0.88 \pm 0.07$ & $1.06 \pm 0.07$ & $1.23 \pm 0.11$ & $1.34 \pm 0.13$ \\
\hline
\end{tabular}

The relationship between average stride length $L_{m}$ (expressed in $\mathrm{m}$ ) and average walking speed $V_{m}$ (expressed in $\mathrm{m} / \mathrm{s}$ ) is analyzed using standard regression tools: $L_{m}=0.46$ $V_{m}+0.77\left(r^{2}>0.99\right)$. The same is done for the relationship between the average stride time $T_{m}$ (expressed in s) and $V_{m}$, thereby obtaining: $T_{m}=-0.65 V_{m}+1.89\left(r^{2}>0.99\right)$.

The average walking speed by inertial dead-reckoning is highly correlated with the estimate of the same quantity produced by GPS $\left(r^{2}>0.98\right)$. The average distance traveled for each track is estimated by inertial dead-reckoning: $L_{\mathrm{IMU}}=$ $401.2 \pm 4.61 \mathrm{~m}($ mean $\pm \mathrm{SD})$ and by GPS: $L_{\mathrm{GPS}}=409.5 \pm$ $10.92 \mathrm{~m}$.

The heading information concerning the direction of displacement can be obtained when GPS moves. Fig. 5 shows the reconstruction of the path corresponding to a $400 \mathrm{~m}$ track obtaining by combining the azimuth from GPS with the displacement from the foot IMU, starting from a point which is conventionally taken as the origin of the ground coordinate frame in a direction corresponding to null azimuth.

The results of a final experiment, where the subject is asked to walk for a $2,000 \mathrm{~m}$ track with no interruption, are reported in Fig. 6.

\section{Discussion AND CONCLUSIONS}

Stride length and time estimated by IMU appear to be characterized by low variability, which is partly due to moderate intra-subject physiological variability at all tested 
cadences, and partly due to the precision of the signal processing method adopted.

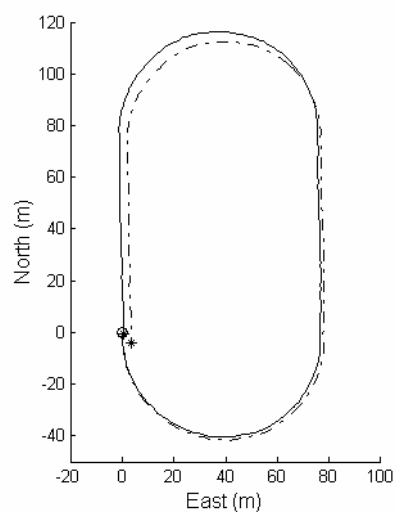

Fig. 5 East-position versus North-position from GPS (solid line) and from inertial dead-reckoning combined with GPS-derived azimuth estimate (dashed-dot line). Start and end positions are indicated using a circle and star, respectively.

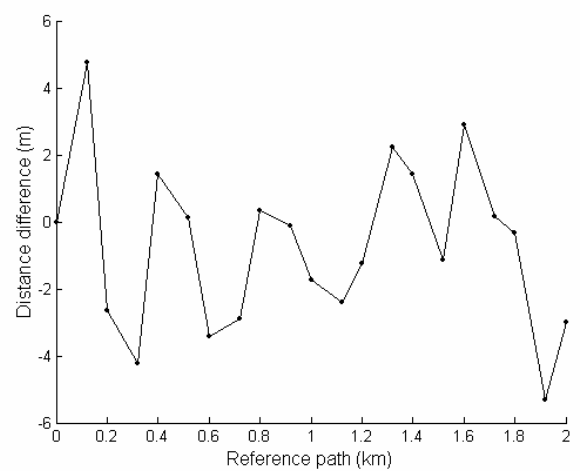

Fig. 6 Difference between traveled distances as indicated by inertial deadreckoning and GPS corresponding to various checkpoints for a $2 \mathrm{~km}$ track.

Interestingly, the statistics in Table 1 are collected from a number of walking trials conducted in several days, with the additional effect on data variability of environment conditions and IMU placement in different days. Further analysis of the data reported in Table 1 allows to confirm the existence of a linear relationship between stride time and walking velocity a feature which is considered basic to the way humans tend to walk in freely-selected conditions [8].

Comparing the traveled distances estimated by IMU and GPS allows to state that inertial dead-reckoning is remarkably accurate (GPS readings are characterized by HDOP $<3$ during our experimental sessions - five-seven satellites in view at all times). Since the IMU mode of operation consists of delivering spatial information using stride time as the basic unit of time, at each checkpoint we have to wait until the next stride occurrence to obtain an update of the traveled distance. In other words, the estimated distance obtained from inertial dead-reckoning can be in error of one stride (worst case) at each checkpoint.

The performance level achieved by foot inertial sensing derives from implementing a variety of auto-nulling and auto- resetting techniques, which help improving the strapdown integration. These techniques are well-suited to deal with quasi-periodical signals, since integrations are limited to intervals lasting just few tenth of second, before alignment and integration are carried out again in preparation for the next gait cycle.

The results of the experimentation are obtained in outdoor conditions, and they basically confirm the results of concurrent work of ours, which aims at analyzing treadmill walking using the same measuring approach [13]. The main limitation of the present approach is the need for reliance on the sagittal model of human gait; significant departures from this model, i.e., as when a human being suddenly changes his direction of walking, may turn out into errors, which can be counteracted either by resorting to more complex IMUs or developing more sophisticated processing, [16].

\section{REFERENCES}

[1] A.M. Sabatini, "Inertial sensing in biomechanics: a survey of computational techniques to bridge between motion analysis and personal navigation," submitted.

[2] E. Foxlin, "Motion tracking requirements and technologies," In Handbook of virtual environments, Stanney K.(Ed.), Lawrence Erlbaum Associates, 2002.

[3] P. Terrier, Q. Ladetto, B. Merminod, and Y. Schutz, "High-precision satellite positioning system as a new tool to study the biomechanics of human locomotion", J. Biomech., vol. 33, pp. 1717-1722, 2000.

[4] C.T. Judd, "A personal dead reckoning module," Proc. ION FPS-97, Kansas City, MO, U.S.A., pp. 47-51, 1997.

[5] Q. Ladetto, and B. Merminod, "In step with INS,", GPS World, 10, pp. 30-38.

[6] R.. Jirawimut, P. Ptasinski, V. Garaj, F. Cecelja, and W. Balachandran, "A method for dead reckoning parameter correction in pedestrian navigation system", IEEE Trans. Instrum. Meas., vol. 52, pp. 209-215, 2003.

[7] R.G. Brown, and P.Y.C. Hwang, Introduction to random signals and applied Kalman filtering, Wiley, New York, 1997.

[8] W. Zijlstra, and A.L. Hof, "Assessment of spatio-temporal parameters from trunk acceleration during human walking," Gait \& Posture, vol 18, pp. 1-10, 2003.

[9] R. Williamson, and B.J. Andrews, "Detecting absolute human knee angle and angular velocity using accelerometers and rate gyroscopes,' Med. Biol. Eng. \& Comp., vol. 39, pp. 294-302, 2001.

[10] K. Aminian, B. Najafi, C. Büla, P.-F. Leyvraz, and Ph. Robert, "Spatiotemporal parameters of gait measured by an ambulatory system using miniature gyroscopes," J. Biomech., vol. 35, pp. 689-699, 2001.

[11] I.P.I. Pappas, M.R. Popovic, T. Keller, V. Dietz, and M. Morari, “A reliable gait phase detection system,” IEEE Trans. Neural Syst. Rehab. Eng., vol. 9, pp. 113-125, 2001.

[12] O. Perrin, P. Terrier, Q. Ladetto, B. Merminod and Y. Schutz, "Improvement of walking speed prediction by accelerometry and altimetry, validated by satellite positioning," Med. Biol. Eng. Comp., vol. 38, pp. 164-168, 2000.

[13] A.M. Sabatini, C. Martelloni, S. Scapellato, and F. Cavallo, "Assessment of walking features from foot inertial sensing," IEEE Trans. Biomed. Eng., vol. 52, no. 3, pp. 486-494, 2005.

[14] A.M. Sabatini, "Wearable sensor systems in biomechanics: assessment of unrestrained walking features," Proc. Internat. Meas. Technol. Conf., 2004, Como, Italy, pp. 881-883.

[15] D. McNeil Mayhew, "Multi-rate sensor fusion for GPS navigation using Kalman filtering," Ms. Thesis, Virginia Polytechnic Institute, May 1999.

[16] A.M. Sabatini, "Quaternion-based strap-down integration method for applications of inertial sensing to gait analysis", Med. Biol. Eng. Comput., vol. 43, no. 1, pp. 94-101, 2005. 